\title{
LES PROTOCOLES ADDITIONNELS AUX CONVENTIONS DE GENËVE EN QUÊTE D'UNIVERSALITÉ
}

Voici dix ans, le 8 juin 1977, la Conférence diplomatique sur la réaffirmation et le développement du droit international humanitaire applicable dans les conflits armés adoptait deux Protocoles additionnels aux Conventions de Genève de 1949 relatifs l'un à la protection des victimes des conflits armés internationaux, l'autre à la protection des victimes des conflits armés non internationaux.

Chacun s'accordait alors à penser que ces nouveaux instruments juridiques constituaient une étape très importante de la codification $d u$ droit international humanitaire parce qu'ils complétaient les dispositions des Conventions de Genève tout en adaptant les normes humanitaires en vigueur aux réalités contemporaines.

Si tant est qu'on pouvait l'espérer, la fréquence des conflits armés n'en a pas été pour autant ralentie, tant s'en faut! Certes, des conflits se sont éteints, mais d'autres ont perduré tandis que de nouvelles guerres éclataient dans toutes les régions du monde.

Aussi, au bout de dix ans, peut-on légitimement se poser la question de savoir si cet effort de codification a eu pour effet, à défaut d'avoir pu réduire le nombre de ces conflits, du moins d'inciter les parties opposées à mieux respecter les règles du droit humanitaire.

Certes, le recul actuel n'est probablement pas suffisant pour dresser un bilan dont les conclusions seraient indiscutables; mais ce dixième anniversaire de l'adoption des Protocoles doit être l'occasion de procéder à une analyse approfondie de l'état actuel de leur ratification, et de s'interroger sur l'attitude des Etats quant au respect du droit humanitaire et sur le rôle de ses agents promoteurs, comme le CICR.

Ces questions importantes sont depuis toujours au ccur des préoccupations du CICR. Elles m'ont inspiré quelques réflexions personnelles que je désire partager avec les lecteurs de la Revue, à l'occasion de mon entrée en fonction comme Président du CICR.

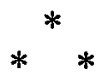


L'élaboration des projets de Protocoles jusqu'à leur adoption a constitué une entreprise plus ardue que la négociation des Conventions de 1949. Le législateur international a dî prendre en compte plusieurs facteurs qui, dès les années 1950, ont profondément modifié le système international: tout d'abord la participation massive de nouveaux Etats indépendants à la vie de la communauté mondiale, qui a transformé le processus de création du droit international; ensuite la prolifération de conflits localisés et de conflits internes, véritable exutoire à la grande peur dissuasive de la guerre nucléaire; enfin, une certaine radicalisation des idéologies qui a favorisé la création de blocs politiques antagonistes tandis que s'accroissait le fossé entre pays riches, industrialisés, certains surarmés, et les pays en développement.

Les Etats restent maîtres du jeu, certes, mais il faut désormais compter aussi avec les mouvements de libération nationale, les guérilleros, les peuples et les individus élevés déjà par certains experts au rang de sujets de droit.

L'action humanitaire a été largement affectée par cette situation. Fondée jusqu'alors sur une certaine conception de la conduite de la guerre, sur des modèles différenciant nettement les combattants de ceux qui ne l'étaient pas, l'action humanitaire pouvait s'exercer normalement en référence aux lois de la guerre. Mais la situation se brouille singulièrement dans les années 1960: la banalisation du mythe de la guerre révolutionnaire parait légitimer aux yeux de certains le recours à tous les moyens de lutte, remettant ainsi en question le droit humanitaire. Comment distinguer véritablement les catégories de victimes? Qui a droit à une protection? Qu'est-ce qu'un prisonnier de guerre?

L'existence et le développement d'armes de destruction massive rendent difficile, voire impossible, la distinction entre civils et militaires et menacent les fondements mêmes du droit humanitaire. Comment garder une dimension humaine dans des guerres devenues totalement destructrices? Comment canaliser ces manifestations de violence qui menacent le fonctionnement du système international? Comment résoudre le désaccord fondamental sur l'illégitimité de principe du recours à la violence et des règles que l'on veut fixer, malgré tout, lors de conflits armés?

Toutes ces questions constituaient autant d'enjeux qui ont largement dominé le déroulement de la Conférence diplomatique de 1974 à 1977.

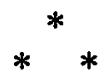


Malgré les efforts de la Confédération suisse, pays hôte, et du CICR, la Conférence diplomatique n'a pu éviter complètement la politisation. Mais on savait que "l'humanitaire n'est pas chimiquement pur" car si la protection est humanitaire, sa mise en auvre soulève des problèmes politiques et militaires. L'histoire se répète: les Protocoles, pas plus que les Conventions de Genève, n'ont supprimé la tension entre les politiques étatiques et les exigences de l'humanité.

Ainsi les Etats sont-ils disposés à protéger davantage de personnes et à mieux réglementer la conduite des hostilités. Ceci est fondamental certes, mais pour autant, pour les uns, qu'on n'entre pas en matière dans la question de la guerre nucléaire, à condition, pour les autres, qu'en cas de conflit interne, on restreigne au maximum la protection des forces dissidentes.

Les pays du tiers monde qui effectuent une entrée en force ont obtenu de faire reconnaître les guerres de libération nationale en tant que conflits internationaux et à élargir la notion de combattant et de prisonnier de guerre pour y inclure, sous certaines conditions, les guérilleros. On se demandait certes, à l'époque déjà, si ces nouvelles dispositions pourraient être acceptées sans problèmes, notamment par les pays directement concernés!

Mais le spectre de la "guerre juste" a été évité, c'est-à-dire la tentation de fixer, dans le droit humanitaire, des règles différenciées en fonction de la cause défendue: une telle approche aurait, à l'évidence, porté un coup fatal au droit humanitaire. Et le consensus s'est établi sur l'essentiel: la protection de la population civile contre les dangers de la guerre indiscriminée, l'interdiction des bombardements massifs ou indiscriminés ou à titre de représailles. A cet acquis fondamental, nous associerons la protection renforcée du personnel, des formations et des transports sanitaires, ainsi que celle de la protection civile, la sauvegarde de l'environnement et des biens culturels et bien évidemment les dispositions adaptées limitant le droit des belligérants de choisir les méthodes et moyens de conduire les opérations militaires.

Peut-on encore douter que la protection de l'individu n'ait été l'un des objectifs primordiaux des Protocoles quand les Etats s'entendent pour renforcer les droits conférés à l'individu et accorder des garanties fondamentales minima de traitement humain à tous les individus en temps de conflits armés, internationaux ou internes?

Enfin un louable effort a été consenti pour renforcer les mécanismes de contrôle et de sanction. 
Et je pense aujourd'hui que la Conférence a finalement adopté, par consensus, rappelons-le, deux bons textes. Fruit de sages compromis, les deux Protocoles renforcent considérablement la protection des victimes des conflits armés.

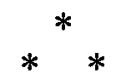

Dix ans après l'adoption des Protocoles, 67 Etats sont Parties au Protocole I et 61 au Protocole II, ce qui représente environ le tiers des Etats parties aux Conventions de Genève.

Il s'agit là d'un bilan qui peut paraître encourageant, mais c'est vraiment trop peu si l'on considère que les instruments de droit international humanitaire ont une vocation universelle. Or cette universalité n'est aujourd'hui pas acquise. Des cinq pays membres permanents du Conseil de Sécurité de l'ONU, par exemple, seule la Chine a ratifié les deux Protocoles, la France seulement le Protocole II. L'hypothèque historique qui pesait, à tort ou à raison, sur les Conventions de Genève du fait de leur caractère euro-centré a été levée mais l'universalité des nouvelles normes du droit humanitaire reste à conquérir.

Reconnaissons-le, les obstacles à l'universalisation du droit humanitaire sont étroitement liés aux motivations politiques et idéologiques qui sous-tendent le droit, aujourd'hui plus que jamais. Dans de nombreux cas, quand il s'agit de construire le droit, chaque Etat s'efforce d'imposer la règle qui épouse de la manière la plus adéquate l'intérêt qu'il entend faire prévaloir. Et quand il faut appliquer le droit, l'interprétation unilatérale de l'Etat continue à l'emporter au nom de la souveraineté. Malheureusement le droit humanitaire n'échappe pas à la règle. Dans cette confrontation entre le pouvoir et le droit, le respect des règles humanitaires reste trop souvent assujetti à des considérations basées sur la souveraineté de l'Etat. En effet, sur le plan de la mise en auvre, les Etats continuent à pêcher par omission - ou par calcul - ; on reconnaît bien volontiers que le droit humanitaire s'est enrichi, qu'il est adapté à tous les conflits armés et qu'il dispose de mécanismes adéquats de mise en ouvre mais l'expérience montre que généralement - hélas! - «on évacue l'humanitaire dans l'accessoire»!

Sans doute les obstacles à l'accession aux Protocoles ainsi que les difficultés d'application du droit humanitaire sont-ils accentués par les réalités internationales plus préoccupantes qu'il y a dix ans: les violations du droit se multiplient à mesure que les guerres locales 
prolifèrent ou s'enfoncent dans la durée et la violence. La stratégie de la "guerre totale» ignore décidément l'humanitaire! Elle nourrit la dégradation inquiétante de la règle de droit. Le CICR, par la voix de mon prédécesseur Alexandre Hay, n'a cessé de dénoncer la violation répétée des principes humanitaires: "Tous les prétextes sont utilisés pour justifier les actions injustifiables: impératifs militaires, sécurité de l'Etat, dernier recours des peuples opprimés".

En vérité, au-delà des arguties juridiques, le problème fondamental de la promotion du droit international humanitaire, et donc également de la ratification des Protocoles, c'est trop souvent le manque de réelle volonté politique des Etats au niveau de l'application du droit.

En adoptant les Conventions de Genève en 1949 et leurs Protocoles en 1977, les Etats se sont engagés non seulement à respecter mais aussi à faire respecter le droit humanitaire. Ce qui signifie qu'ils engageaient leurs responsabilités non seulement pour des violations dont ils seraient coupables, mais aussi pour celles des autres gouvernements parties aux Protocoles. A bien réfléchir, cette clause tranche par son audace! Mais quel étrange paradoxe entre cette acceptation par les Etats d'obligations humanitaires étendues et leur prudence, voire leur réticence à les mettre en pratique et de façon générale entre cette adhésion aux valeurs humanitaires et le manque de rigueur dans la volonté politique de les défendre! Or, ces valeurs humanitaires sont des sources matérielles du droit humanitaire qui repose sur la volonté des Etats. On ne citera jamais assez un autre de mes prédécesseurs, Max Huber, qui affirmait: "Du point de vue strictement juridique, un véritable droit de l'Humanité s'est créé en vertu duquel la personne humaine, son intégrité, sa dignité, sont défendues au nom d'un principe moral qui s'élève bien au-delà des limites du droit national et de la politique».

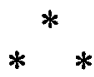

Le défi de ces prochaines décennies sera de tenter de réconcilier les valeurs humanitaires et la volonté politique des Etats, autrement dit d'établir la complémentarité entre ces valeurs et la souveraineté étatique afin que les Etats respectent pleinement et volontairement ces valeurs comme principes de référence dans leurs politiques et leurs actions. Il s'agit en fait de mobiliser la communauté des Etats afin d'accroître le réflexe humanitaire dans la prise de décision politique. 
Cette mobilisation humanitaire dispose d'un arsenal impressionnant de règles qui répondent à l'essentiel; elle doit aussi inclure un appel aux valeurs traditionnelles, religieuses et éthiques, à l'intérêt politique et économique des Etats; elle doit pouvoir gagner le concours de l'opinion publique.

Telle est la conviction du CICR, maintes fois rappelée ces derniers mois et plus récemment lors de la $X X V^{e}$ Conférence internationale de la Croix-Rouge qui s'est adressée solennellement aux Etats non encore Parties aux Protocoles pour les inviter à les ratifier ou à y adhérer "le plus rapidement possible».

Pour sa part, le CICR continuera à encourager les Etats à accéder aux Protocoles jusqu'à ce qu'ils soient acceptés universellement. Mais il est souhaitable, sinon impératif, que ses efforts soient largement appuyés. Déjà, nous nous réjouissons de cette prise de conscience qui a conduit les Nations Unies, plusieurs organisations régionales et des institutions internationales à recommander la ratification des Protocoles et à appuyer les efforts du CICR dans son action.

Le CICR sera en effet en meilleure position pour inciter les Etats à ratifier les Protocoles et à en réclamer publiquement l'application s'il n'est pas seul à le faire. Des Etats non engagés dans un conflit peuvent servir de relai. Rappelons tout particulièrement le rôle de la Suisse qui, en sa qualité de dépositaire des Conventions de Genève, a su faire entendre sa voix au caur de conflits tels que celui entre l'Iran et l'Irak ou celui du Liban pour que l'application du droit humanitaire soit universelle, quels que soient l'origine des conflits et les motifs idéologiques des Parties. Cela avait été déjà le cas pendant la Conférence diplomatique de 1974-77 pour laquelle les autorités suisses avaient su mettre à la disposition des Etats le cadre propice à la négociation des Protocoles. La politique de neutralité, permanente et armée, de la Suisse, son expérience de puissance protectrice pendant la Seconde Guerre mondiale (et à de trop rares occasions depuis les années 1950), et son sens de la mesure lui confèrent une autorité reconnue de tous. Fidèle à cette ligne de conduite, la Suisse continuera - je l'espère - à entreprendre des efforts particuliers pour faire. accepter les Protocoles par les Etats. Le CICR saisit l'occasion du $X^{e}$ anniversaire de l'adoption des Protocoles pour lui exprimer sa gratitude.

Enfin, les Sociétés nationales de la Croix-Rouge et du CroissantRouge dont le rôle a été particulièrement renforcé dans les Protocoles peuvent continuer à agir efficacement auprès de leurs gouvernements dans ce processus de ratification et d'adhésion aux Protocoles comme 
dans les programmes de diffusion du droit. Nous sommes particulièrement heureux, à l'occasion de ce dixième anniversaire de l'adoption des Protocoles d'ouvrir les colonnes de la Revue à certaines d'entre elles.

La ratification des Protocoles, le respect du droit international humanitaire nous concernent tous. Il est vital que les Etats parties aux Conventions de Genève respectent et fassent respecter le droit international humanitaire, il est primordial que les Protocoles deviennent universels. Comme l'a dit le professeur Pictet, "il y va de la survie de l'humanité".

Mais au-delà du respect de l'ennemi tombé et du civil innocent, qui est l'essence même du droit humanitaire, nous voulons voir dans l'engagement du Mouvement international de la Croix-Rouge et du Croissant-Rouge en faveur des Protocoles, une action concrète vers l'silification d'un monde de paix!

Cornelio Sommaruga Président du CICR 